\title{
Individual Differences in Reward Drive Predict Neural Responses to Images of Food
}

\author{
John D. Beaver, ${ }^{1}$ Andrew D. Lawrence, ${ }^{1}$ Jenneke van Ditzhuijzen, ${ }^{1}$ Matt H. Davis, ${ }^{1}$ Andrew Woods, ${ }^{2}$ and \\ Andrew J. Calder ${ }^{1}$ \\ ${ }^{1}$ Medical Research Council Cognition and Brain Sciences Unit, Cambridge CB2 2EF, United Kingdom, and ${ }^{2}$ School of Psychology, University of Wales, \\ Bangor, Gwynedd LL57 2AS, United Kingdom
}

\begin{abstract}
A network of interconnected brain regions, including orbitofrontal, ventral striatal, amygdala, and midbrain areas, has been widely implicated in a number of aspects of food reward. However, in humans, sensitivity to reward can vary significantly from one person to the next. Individuals high in this trait experience more frequent and intense food cravings and are more likely to be overweight or develop eating disorders associated with excessive food intake. Using functional magnetic resonance imaging, we report that individual variation in trait reward sensitivity (as measured by the Behavioral Activation Scale) is highly correlated with activation to images of appetizing foods (e.g., chocolate cake, pizza) in a fronto-striatal-amygdala-midbrain network. Our findings demonstrate that there is considerable personality-linked variability in the neural response to food cues in healthy participants and provide important insight into the neurobiological factors underlying vulnerability to certain eating problems (e.g., hyperphagic obesity).
\end{abstract}

Key words: reward; personality; appetite; striatum; amygdala; orbitofrontal cortex

\section{Introduction}

Physiological states associated with energy balance (e.g., hunger and satiety) are major determinates of eating behavior. However, food selection and intake are also powerfully influenced by the rewarding properties of individual items, such as their taste, smell, and sight (Toates, 1981; Berridge, 1996; Saper et al., 2002). Indeed, exposure to highly appetizing food cues can override satiety signals and promote overeating (Cornell et al., 1989). Comparative research has implicated a network of interconnected brain regions comprising frontal, ventral striatal, amygdala, and midbrain regions in aspects of food reward (Berridge, 1996, 2003; Ikemoto and Panksepp, 1999; Kelley, 2004; Balleine, 2005; Di Chiara, 2005; Kelley et al., 2005). Direct pharmacological stimulation of this network produces hyperphagia and preferentially increases the intake of foods high in fat and sugar, even in animals fed to apparent satiety (Kelley, 2004; Kelley et al., 2005).

Human brain imaging studies have shown that visual cues associated with appetizing food stimuli (including pictures of foods) engage components of this reward network (LaBar et al., 2001; Morris and Dolan, 2001; Gottfried et al., 2002; O’Doherty et al., 2002; Killgore et al., 2003; Wang et al., 2004), consistent with the notion that such cues can acquire motivating and hedo-

Received Jan. 25, 2006; revised March 21, 2006; accepted March 22, 2006.

This work was supported by The Medical Research Council (UK). MRI scanning was performed at the University of Cambridge Wolfson Brain Imaging (entre (WBIC). We thank Jill Keane for assistance with data collection, Rik Henson for helpful advice, Brian Cox for graphics support, and the radiographers of the WBIC.

Correspondence should be addressed to either Dr. John Beaver or Dr. Andrew Calder, Medical Research Council Cognition and Brain Sciences Unit, 15 Chaucer Road, Cambridge CB2 2EF, UK. E-mail: john.beaver@mrc-cbu. cam.ac.uk; andy.calder@mrc-cbu.cam.ac.uk.

DOI:10.1523/JNEUROSCI.0350-06.2006

Copyright $\odot 2006$ Society for Neuroscience $\quad$ 0270-6474/06/265160-07\$15.00/0 nic properties themselves (Cardinal et al., 2002). An implicit assumption of this research is that all healthy individuals respond similarly to appetizing or pleasurable stimuli. However, sensitivity to rewarding stimuli can vary substantially from one individual to the next (Gray, 1987). Individual differences in measures of trait reward sensitivity predict food cravings, hyperphagia, and relative body weight (Davis et al., 2004; Dawe and Loxton, 2004; Franken and Muris, 2005). Despite these connections, and widespread theorizing that reward sensitivity reflects reactivity in a ventral striatal-amygdala-midbrain system (Depue and Collins, 1999; Pickering and Gray, 1999), it remains to be determined how variability in this trait is expressed at the neurobiological level. Here we report the first demonstration of individual differences in neural responses to pictures of appetizing foods related to variation in trait reward drive (i.e., the tendency to pursue reward).

We used functional magnetic resonance imaging (fMRI) to measure blood oxygen level-dependent (BOLD) response while participants passively viewed images of highly palatable, appetizing foods (e.g., chocolate cake, ice cream). Comparison conditions included bland foods (e.g., uncooked rice, potatoes) and nonfood objects (e.g., videocassette, iron). Because some brain regions implicated in reward (e.g., orbitofrontal cortex, amygdala, and ventral striatum) have also been associated with processing aversive stimuli (Heining et al., 2003; Jensen et al., 2003; Zald, 2003; Kringelbach and Rolls, 2004), we also included an aversive food category comprising pictures of disgusting foods (e.g., rotten meat, moldy bread). Reward drive was assessed with the Behavioral Activation Scale (BAS)-drive scale (Carver and White, 1994), which measures an individual's general tendency to actively pursue reward. We focused our analyses on regions previously implicated in responding to visual cues of food re- 
Table 1. Ratings of stimuli used in the experiment

\begin{tabular}{llllll}
\hline & \multicolumn{4}{l}{ Mean stimulus ratings } & \\
\cline { 2 - 6 } & Nauseating & Disgusting & Appetizing & Pleasant & Arousing \\
\hline Appetizing foods & $1.42 \pm 0.51$ & $1.26 \pm 0.27$ & $5.36 \pm 0.59$ & $4.75 \pm 0.98$ & $4.25 \pm 1.23$ \\
Bland foods & $1.40 \pm 0.52$ & $1.29 \pm 0.40$ & $3.16 \pm 0.72$ & $3.43 \pm 1.14$ & $3.21 \pm 1.11$ \\
Disgusting foods & $4.91 \pm 1.32$ & $4.38 \pm 1.54$ & $1.19 \pm 0.17$ & $1.46 \pm 0.44$ & $4.56 \pm 1.50$ \\
Nonfood objects & & $1.13 \pm 0.18$ & & $3.11 \pm 1.23$ & $3.54 \pm 1.20$ \\
\hline
\end{tabular}

Mean \pm SD ratings indicating the degree to which stimuli used in the experiment were pleasant, disgusting, and arousing and the degree to which food stimuli were appetizing and nauseating.

ward: the ventral striatum, amygdala, orbitofrontal cortex, ventral pallidum, and midbrain regions associated with high dopamine and opioid concentrations (i.e., substantia nigra and ventral tegmental areas).

\section{Materials and Methods}

Participants. Fourteen right-handed, healthy volunteers with normal vision and no past neurological or psychiatric history participated in the study for payment. The study was approved by the National Health Service Local Research Ethics Committee for Cambridge, and all participants provided written informed consent before taking part. Excessive movement in the scanner meant that two of the participants' data had to be discarded, leaving seven females and five males, with a mean \pm SD age of $22 \pm 2.4$ years. Participants were restricted from eating for $2 \mathrm{~h}$ before the testing session.

Before scanning, participants completed the Behavioral Inhibition Scale (BIS)/BAS (Carver and White, 1994), a questionnaire that assesses three personality measures related to reward sensitivity (BAS) and one related to behavioral inhibition/anxiety (BIS). The BAS scales include items tapping strong pursuit of appetitive goals (BAS drive) (e.g., "I go out of my way to get things I want"), the inclination to seek out new rewarding situations (BAS fun seeking) (e.g., "I'm always willing to try something new if I think it will be fun"); and positive affect/excitability (BAS reward responsiveness) (e.g., "When good things happen to me, it affects me strongly"). According to Dawe and colleagues (Dawe et al., 2004; Dawe and Loxton, 2004), BAS drive is a clear measure of appetitive motivation and approach behavior and is purported to closely reflect individual differences in the activity of ventral-striatal-related circuitry (Pickering and Gray, 1999). Relative to the other BAS scales, it is the best predictor of positive affective responses to cues of impending reward, has a unique predictive quality to such cues over and above that offered by the other two scales, and has the highest internal reliability (Carver and White, 1994; Jorm et al., 1998). Consistent with these observations, in the current study, BAS drive constituted the most effective predictor of neural activity in brain reward regions, and we focus on this measure. Although some research has used a BAS "total" score, structural equation modeling has shown that the three BAS measures do not form a unitary global measure of appetitive motivation and should be treated as separate scales (Ross et al., 2002). The BIS scale was designed to assess sensitivity to punishment. An example BIS item is, "If I think something unpleasant is going to happen, I usually get pretty worked up."

Questionnaire scores for our sample were as follows: BAS drive, range of 7-13, mean $\pm \mathrm{SD}$ of $10.3 \pm 1.9$; BAS fun seeking, range of $10-15$, mean \pm SD of $13.1 \pm 1.6$; BAS reward responsiveness, range of 15-20, mean \pm SD of $17.6 \pm 1.4$; and BIS, range of $19-25$, mean \pm SD of $21.0 \pm 1.9$.

Stimuli and design. During scanning, participants passively viewed full color photographs of foods consistently identified as highly appetizing (e.g., chocolate cake, ice cream sundae), disgusting (e.g., rotten meat, moldy bread), or bland (e.g., uncooked rice, potatoes), and similar photographs of nonfood objects (e.g., videocassette, iron). There were 30 exemplars in each class. Nonfood images consisted of visually complex objects requiring similar perceptual analysis to the food stimuli but lacking any obvious affective/motivational value. Stimuli were selected from pilot ratings $(n=12)$ made by a different group of individuals to those that participated in the fMRI study. Ratings were made on a Likert scale (from 1 to 7 ) and indicated the degree to which each stimulus was pleasant, disgusting, and arousing and the degree to which each food stimulus was appetizing and nauseating. Mean ratings for the stimuli used are shown in Table 1.

Wilcoxon's signed rank tests showed that appetizing foods were rated as significantly more pleasant than each of the other categories ( $p$ values $<0.005)$ and as more appetizing than disgusting and bland foods ( $p$ values $<0.005$ ). Disgusting foods were rated as significantly more disgusting than each of the other categories ( $p$ values $<0.005$ ) and more nauseating than pleasant and bland foods ( $p$ values $<0.005)$. None of the stimulus categories differed significantly on ratings of arousal ( $p$ values $>0.05$ ). During the fMRI experiment, stimuli were presented in alternating $24 \mathrm{~s} \mathrm{blocks.} \mathrm{Each}$ block contained 10 images from the same category (appetizing, disgusting, bland, or nonfood), with a total of six blocks of each type presented in one of two pseudorandom orders. Each image was displayed for 1400 $\mathrm{ms}$, followed by a $1000 \mathrm{~ms}$ interstimulus interval. Stimuli were viewed via an angled mirror above the participants' eyes, which reflected images back-projected from a translucent screen positioned in the bore of the magnet to the rear of the participants' head.

Imaging and statistical analysis. MRI scanning was performed on a 3 tesla Medspec scanner (Bruker, Ettlingen, Germany) with a head coil gradient set. Whole-brain data were acquired with $\mathrm{T} 2{ }^{*}$-weighted echoplanar imaging (EPI), sensitive to BOLD signal contrast (21 interleaved 4-mm-thick slices; $1 \mathrm{~mm}$ interslice gap; repetition time, $3020 \mathrm{~ms}$; acquisition time, $2300 \mathrm{~ms}$; echo time, $27 \mathrm{~ms} ; 90^{\circ}$ flip angle; $25 \times 25 \mathrm{~cm}$ field of view; $64 \times 64$ matrix; $100 \mathrm{kHz}$ voxel bandwidth). Slice acquisition was transverse oblique, angled to avoid the eyeballs. Images were acquired in one run of $\sim 10 \mathrm{~min}$ for each participant. The first six volumes were discarded to allow for equilibration effects.

Data were analyzed using SPM 99 software (Wellcome Department of Imaging Neuroscience, London, UK). EPI images were corrected for slice timing and head movement, and undistortion based on field strength derived from a phase map was applied (Cusack et al., 2003). Masked normalization to a standard EPI template was performed using $2 \mathrm{~mm}$ isotropic voxels (Brett et al., 2001), and images were smoothed with a Gaussian kernel of $8 \mathrm{~mm}$ full-width half-maximum. Condition effects were estimated for each participant at each voxel using boxcar regressors convolved with a canonical hemodynamic response function in a general linear model, with spatial realignment parameters included as regressors to account for residual movement-related variance. A high-pass filter was used to remove low-frequency signal drift, and the data were also lowpass filtered with the canonical hemodynamic response function. Activation contrasts between conditions were estimated for each participant at each voxel, producing statistical parametric maps. Random-effects analysis was conducted to analyze data at a group level, with modulations by individual differences being assessed by separate simple regressions against BAS drive, BAS reward responsivity, BAS fun seeking, and BIS scores.

A priori regions of interest (ROIs) were determined based on areas activated by cues of food rewards in previous studies (i.e., the ventral striatum, amygdala, midbrain regions, ventral pallidum, and orbitofrontal cortex). A statistical threshold of $p<0.001$ uncorrected is used for these a priori ROIs, in line with a number of previous functional imaging investigations of the neural correlates of food reward (O'Doherty et al., 2002; Gottfried et al., 2003). When ROIs corresponded to discreet anatomical structures, we used small volume correction (SVC) for multiple comparisons applied at $p<0.05$. For this purpose, the amygdala and pallidum were defined using structural templates derived by automated anatomic labeling (Tzourio-Mazoyer et al., 2002). For the ventral striatum and midbrain, radius $10 \mathrm{~mm}$ spheres were defined on the basis of a previous study examining responses to visual cues of impending taste rewards (O'Doherty et al., 2002). Anatomical labeling of activations was checked with reference to the atlases of Duvernoy $(1995,1999)$ by overlaying activations onto the mean normalized structural T1 image across participants. For visualizing activations, group maps are overlaid on the ICBM 152 structural template, an average T1-weighted image of 152 individuals coregistered to Montreal Neurological Institute (MNI) space. 
Activations are reported using $(x, y, z)$ coordinates in MNI standardized space.

\section{Results}

\section{Appetizing foods}

We first established group-average neural responses to viewing appetizing foods by performing random-effects contrasts. For the a priori ROIs, increased activation was found in the left anterolateral orbitofrontal cortex when responses to appetizing foods were contrasted with responses to viewing nonfood objects (Fig. 1) (MNI coordinates $-38,50,-8 ; T=4.26, p<0.001$ uncorrected) and disgusting foods $(-36$, $42,-10 ; T=4.21, p<0.001$ uncorrected). Significant activation was also found bilaterally in the ventral striatum when responses to appetizing foods were contrasted with responses to bland foods (Fig. 2) (right, $8,10,-12, T=8.04, p<0.0005$ SVC; left, $-8,16,-12, T=3.98, p<0.05$ SVC). Other brain regions that showed group-average effects for contrasts comparing appetizing foods with each of nonfood objects, disgusting foods, or bland foods (at $p<0.001$ uncorrected) are summarized in supplemental Table 3 (available at www.jneurosci.org as supplemental material).

We then examined the relationship between trait reward drive and neural responses to appetizing food images by entering participants' BAS-drive scores as regressors in simple general linear models fitted to the changes in BOLD signal at each voxel. In line with our expectations, BAS-drive scores significantly predicted activation to appetizing relative to bland foods in all five a priori regions implicated in reward: right ventral striatum $(14,8$, $-18 ; T=4.27, p<0.05$ SVC, $r=0.80, p<$ $0.001)$; left amygdala $(-20,-2,-12 ; T=$ 3.43, $p<0.05$ SVC, $r=0.74, p<0.01)$; substantia nigra/ventral tegmental areas of the midbrain $(0,-20,-28, T=4.38, p<$ 0.05 SVC, $r=0.81, p<0.001$; and $-2,12$, $-20, T=3.82, p=0.08$ SVC, $r=0.77, p<$ 0.005 ); left orbitofrontal cortex (posterior, $-26,16,-24, T=4.36, p<0.001$ uncorrected, $r=0.81, p<0.001$; and anterior, $-34,36,-20, T=4.06, p<0.001$ uncorrected, $r=0.79, p<0.002)$; and left ventral pallidum $(-18,-4,-6 ; T=4.77, p<0.02$ SVC, $r=0.83$, $p<0.001$ ). Additional evidence of midbrain modulation by reward drive was found when appetizing foods were contrasted with nonfood objects $(4,-14,-6 ; T=5.54, p<0.02$ SVC, $r=$ $0.87, p<0.001)$. As shown in Figure 3, activation to viewing pictures of appetizing foods increased as a function of reward drive (BAS drive) in each of these regions.

The BAS-fun-seeking measure showed no significant correlations with activation to appetizing foods in any brain regions. The lack of any significant correlations with fun seeking may relate to
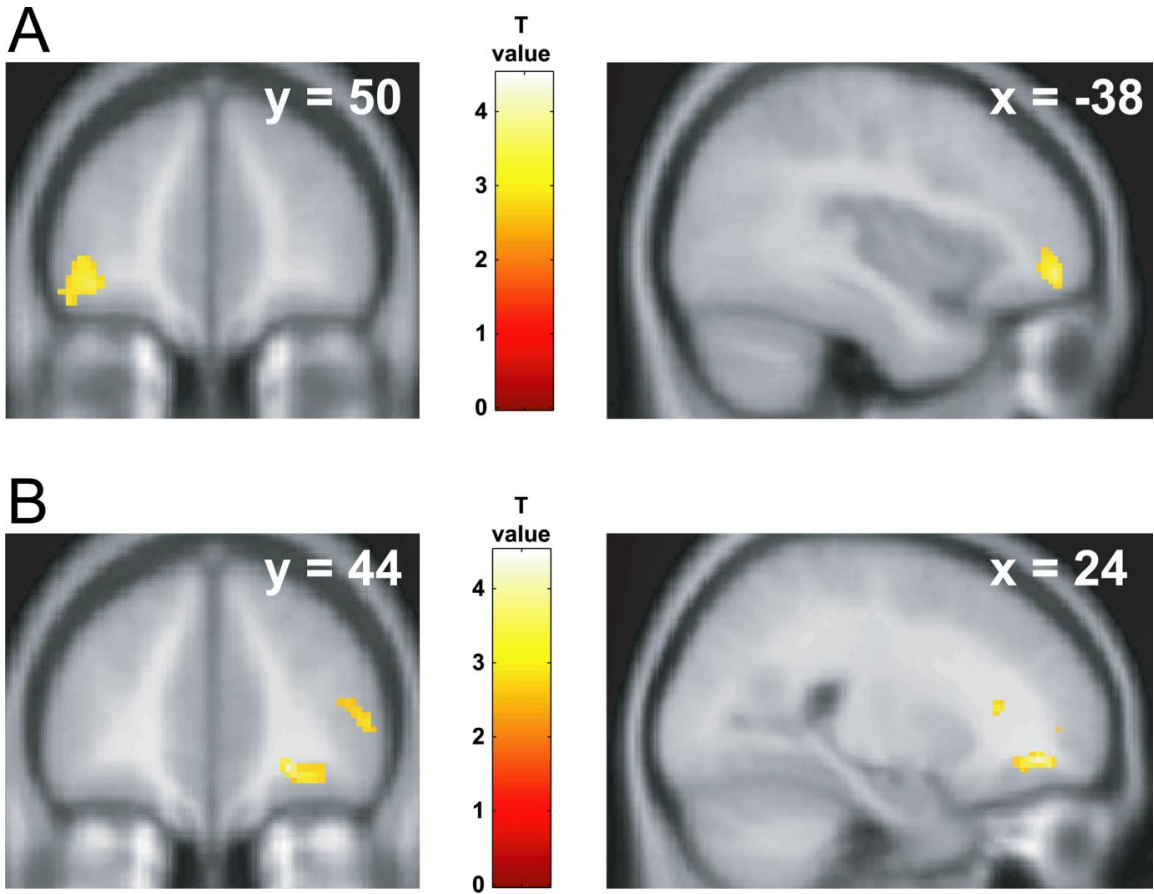

Figure 1. Orbitofrontal responses to viewing appetizing and disgusting foods. A, Area of left orbitofrontal cortex showing increased group-average activation for appetizing foods relative to nonfood objects. $\boldsymbol{B}$, Area of right orbitofrontal cortex showing increased group-average activation for disgusting foods relative to nonfood objects. Color bars indicate $T$ values. Activations are thresholded at $p<0.005$, uncorrected for visualization.
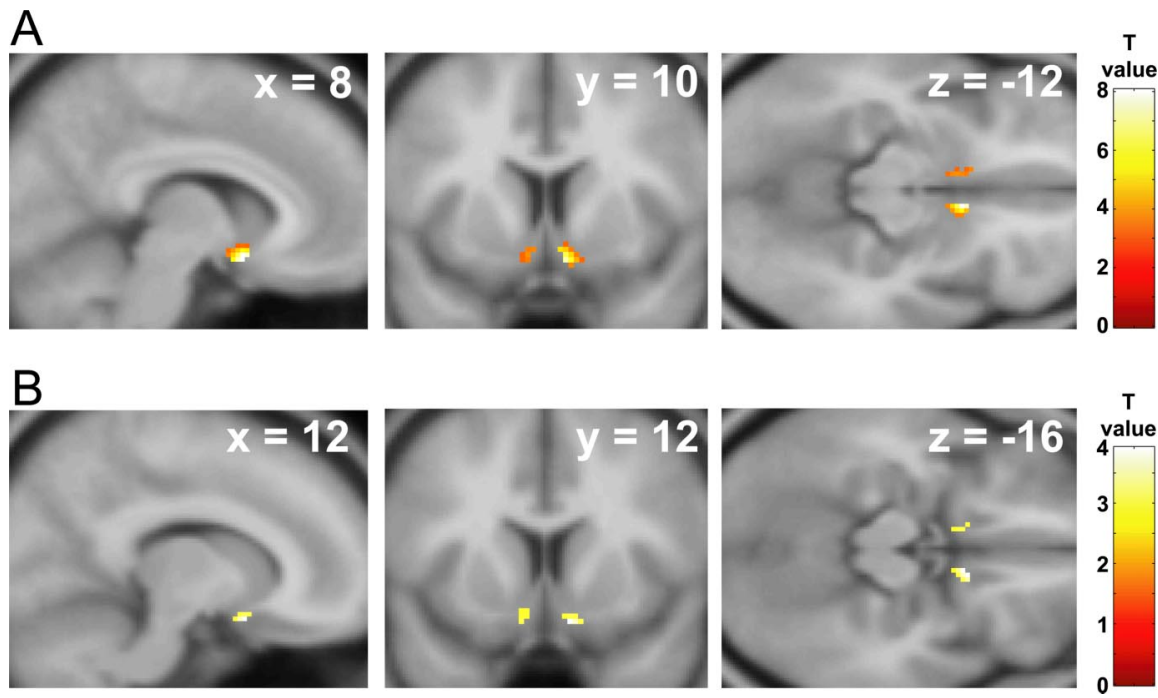

Figure 2. Ventral striatum responses to viewing appetizing and disgusting foods. Areas of ventral striatum showing increased group-average activation for appetizing foods relative to bland foods $(\boldsymbol{A})$ and disgusting foods relative to bland foods $(\boldsymbol{B})$. Color bars indicate $T$ values. Activations are thresholded at $p<0.005$, uncorrected for visualization.

the fact that this measure reflects the inclination to seek out new rewarding experiences as opposed to behavior in response to reward cues in the immediate environment. BAS reward responsiveness produced a significant correlation with activation to appetizing (relative to disgusting) foods in the right medial orbitofrontal cortex $(22,34,-16 ; T=7.30, p<0.001$ uncorrected, $r=0.84, p<0.001)$ and right ventral pallidum $(26,-2,2$; $T=4.30, p<0.05$ SVC, $r=0.80, p<0.001)$. Thus, BAS drive provides the clearest predictor of BOLD response to appetizing foods.

To exclude the possibility that correlations in these areas re- 

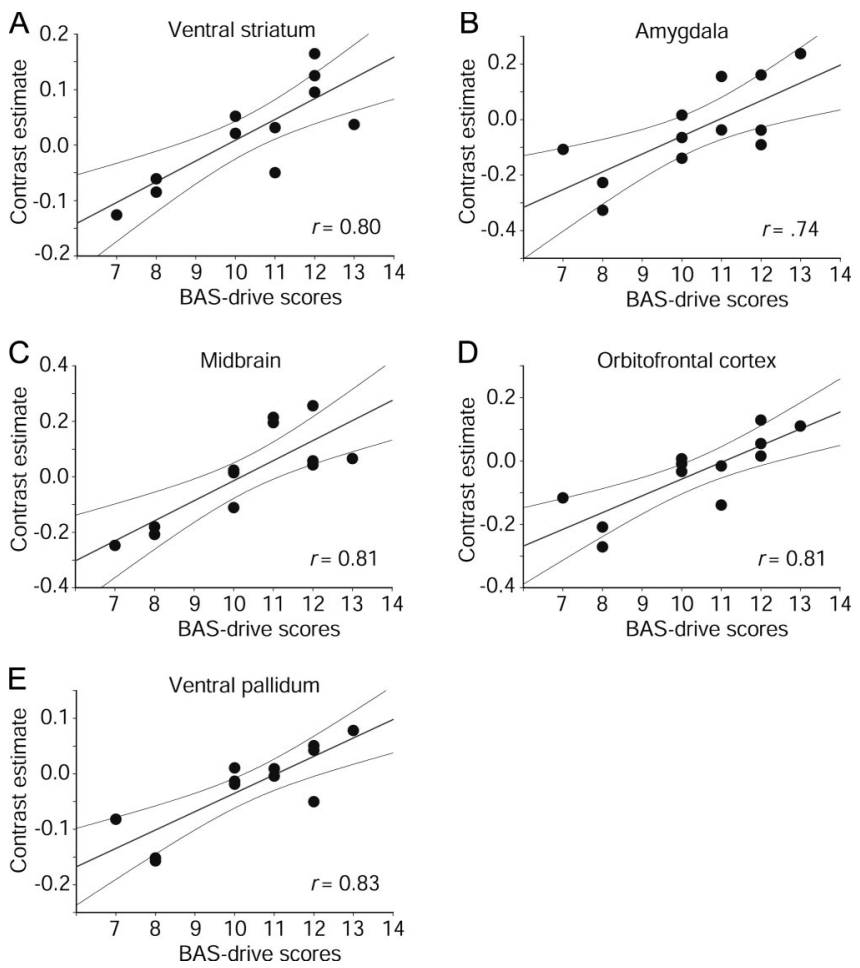

Figure 3. Significant modulation of neural responses to appetizing foods by trait reward drive. Scatter plots show BOLD signal change in peak activated voxels for appetizing relative to bland foods, plotted as a function of participants' BAS-drive scores. Regression lines and $95 \%$ confidence intervals are shown.

flect more general effects related to individual differences in trait arousal/emotionality per se, we examined whether BOLD signal change for appetizing foods relative to each of the other stimulus categories was positively correlated with participants' BIS scores. No significant correlations were found in the a priori ROIs. Thus, the correlations between BAS-drive scores and BOLD response to appetizing foods are unlikely to reflect individual differences in emotional responsivity or arousal more generally.

\section{Disgusting foods}

Group-average subtraction contrasts comparing responses to disgusting foods with each of the other conditions showed significant activation in the right orbitofrontal cortex (Fig. 1) (disgusting vs nonfood objects, $24,44,-8, T=4.51, p<0.001$ uncorrected; disgusting vs bland foods, $16,26,-14, T=4.42, p<0.001$ uncorrected; and disgusting vs appetizing foods, $22,36,-14, T=$ $4.04, p<0.001$ uncorrected) and the right ventral striatum (Fig. 2) (disgusting vs bland foods, 12, 12, $-16 ; T=3.91, p<0.05$ SVC). Other regions responsive to disgusting foods (at $p<0.001$ uncorrected) are summarized in supplemental Table 4 (available at www.jneurosci.org as supplemental material). Included in these areas was the anterior insula, a region implicated in the recognition and experience of disgust (Calder et al., 2001).

As for the appetizing foods, we performed simple regression analyses to investigate whether BOLD response to disgusting foods relative to each of the other conditions showed a significant linear relationship with participants' BAS-drive scores. These analyses indicated a significant correlation in the right orbitofrontal cortex (disgusting vs bland foods, $18,28,-14 ; T=6.07$, $p<0.001$ uncorrected, $r=0.89, p<0.001)$ and the left ventral striatum (disgusting vs bland foods, $-12,12,-16 ; T=3.82, p<$ 0.05 SVC, $r=0.77, p<0.005$ ). In both of these regions, activa- tion to disgusting foods increased as a function of participants' BAS-drive scores.

We also performed simple regressions to investigate whether BOLD signal change for disgusting foods relative to each of the other stimulus categories was correlated with scores from the other scales. No significant correlations were identified in any of our ROIs. Thus, the correlations between BAS-drive scores and neural responses to disgusting foods also appear unlikely to reflect individual differences in general trait emotional responsivity.

\section{Bland foods}

For regions engaged by both the appetizing versus bland and disgusting versus bland contrasts (i.e., ventral striatum, orbitofrontal cortex), it is possible that the change in BOLD response reflects a contribution from one or both of two sources: (1) increased activation to each of appetizing and disgusting foods or (2) decreased activation to bland foods. Similarly, significant correlations with BAS drive could reflect modulation of responses to the appetizing and disgusting or bland foods. Given that different areas of orbitofrontal cortex were sensitive to appetizing and disgusting foods (left for appetizing, right for disgusting), this explanation seems unlikely for this region. However, to fully investigate whether the response to bland foods formed the basis of any of the effects, we conducted an additional analysis in which we contrasted nonfood objects with bland foods. Hence, increased activation for this contrast would reflect decreased activation to bland foods relative to the nonfood baseline. The results showed no significant effects in our hypothesized regions or significant correlations when this contrast was regressed against participants' BAS-drive scores. Therefore, the results of the contrasts comparing each of appetizing and disgusting foods to bland foods do not appear to reflect changes in activation to bland foods alone. In addition, a bland foods versus nonfoods contrast showed no correlations with BAS-drive scores in our regions of interest, demonstrating that the effects we observed are restricted to appetizing and disgusting foods.

For more information, the on-line supplementary materials report all activations showing correlations with BAS drive (supplemental Tables 1, 2, available at www.jneurosci.org as supplemental material), as well as all group-average activations (supplemental Tables 3, 4, available at www.jneurosci.org as supplemental material).

\section{Discussion}

As predicted, we found that individual differences in trait reward drive were strongly correlated with activation to pictures of appetizing foods in a neural network, including ventral striatal, amygdala, midbrain, orbitofrontal, and ventral pallidal regions (Fig. 3) (Öngür and Price, 2000; Kelley, 2004; Fudge et al., 2005; Kelley et al., 2005). Previous functional imaging and comparative research has implicated all five of these areas in food reward (Berridge, 1996; Cardinal et al., 2002; Kelley and Berridge, 2002; Kelley, 2004; Kringelbach and Rolls, 2004; Kelley et al., 2005). Of particular relevance, direct pharmacological activation of this network produces hyperphagia and increases preferentially the intake of foods high in fat and sugar, even in animals fed beyond apparent satiety (Petrovich et al., 2002; Kelley, 2004).

Human behavioral data demonstrate that levels of reward drive and related constructs predict relative body weight (in both normal and overweight populations), food cravings, and hyperphagia, central factors in the etiology and severity of obesity, bulimia nervosa, and binge-eating disorder (Davis and Woodside, 2002; Bulik et al., 2003; Davis et al., 2004; Dawe and Loxton, 
2004; Franken and Muris, 2005). Hence, the present observation that variation in reward drive predicts activation to appetizing food cues in a fronto-striatal-amygdala-midbrain network provides the necessary data to bridge the gap between the human behavioral findings and comparative neurobiology demonstrating a powerful role for this network in motivating food selection and intake. In other words, our data suggest that heightened responsivity of this network to food cues is a mechanism for translating reward drive into increased vulnerability to compulsive-eating disorders in certain individuals. The fact that our study used pictures of foods has additional pertinence to understanding the current high prevalence of these disorders (Abelson and Kennedy, 2004), because such images are widely used in modern society to promote food selection and intake (e.g., advertising, product packaging, vending machines). Indeed, recent findings show that the visual sensory modality can be particularly potent in relation to food cravings (Tiggemann and Kemps, 2005).

In addition to the fronto-striatal-amygdala-midbrain network, variation in reward drive was correlated with activation in the ventral pallidum. The pallidum has seldom been reported in functional imaging studies of reward (Elliott et al., 2000), but comparative research has shown that this area is an important component of the neural systems underlying food motivation and hedonics (McAlonan et al., 1993; Pecina and Berridge, 2000; Tindell et al., 2004; Smith and Berridge, 2005).

Comparative studies have demonstrated the importance of both the appetizing/palatable properties of food-related stimuli and state motivation levels (e.g., hunger) in activating the neural circuits underlying food selection and intake (Berridge, 1996; Bassareo and Di Chiara, 1999; Kelley and Berridge, 2002; Saper et al., 2002). Similarly, human functional imaging investigations have used hunger/satiety manipulations to demonstrate that motivational state can influence reward-related activations in homologous areas (LaBar et al., 2001; Morris and Dolan, 2001; Small et al., 2001; Gottfried et al., 2003; Hinton et al., 2004; Wang et al., 2004). Our current study, however, is the first to show that activation in fronto-striatal-amygdala-midbrain reward circuitry is also related to trait motivation levels. It is worth emphasizing that, although the group-average and regression analyses identified similar regions in the orbitofrontal cortex and ventral striatum, the fronto-striatal-amygdala-midbrain network was only fully identified by regression analyses with BAS drive. This serves to illustrate how variation in this trait can affect the extent to which this network is engaged and the importance of including this dimension as a factor in future research. The fact that regions such as the amygdala were only engaged in individuals with high BAS-drive scores is also consistent with previous research demonstrating that amygdala activation to appetizing food images is only observed in subjects with enhanced state motivation (i.e., in individuals who are hungry) (LaBar et al., 2001).

It is also noteworthy that the correlation between BAS drive and fronto-striatal-amygdala-midbrain activation did not reflect a general alteration in perceived pleasantness of the food items. If this were the case, then we would have expected comparable variability in the neural response to bland foods [because these were both mildly pleasant and mildly appetizing (Table 1)], and consequently the appetizing versus bland contrast would not have correlated with BAS drive. Moreover, a simple variation in perceived pleasantness of the appetizing foods does not appear to capture fully the pattern we observed. Of the regions identified by the regressions against BAS-drive scores, only the orbitofrontal cortex has been shown to correlate with individual differences in subjective pleasantness ratings of food rewards in previous functional imaging studies (Anderson et al., 2003; de Araujo et al., 2003; Kringelbach et al., 2003; Rolls et al., 2003). In this respect, it is interesting that Berridge and colleagues (Berridge, 1996; Berridge and Robinson, 2003; Smith and Berridge, 2005) have distinguished between hedonic and motivational aspects of reward in their comparative work, with the former relating to orbitofrontal and pallidal systems and the latter to dopaminergic ventral striatal and amygdala systems. In line with this distinction, our regression analyses with both BAS drive and BAS reward responsiveness identified the orbitofrontal cortex and ventral pallidum, whereas the full fronto-striatal-amygdala-midbrainpallidal network was only correlated with BAS drive. This is consistent with the fact that the BAS-reward-responsiveness scale taps positive hedonic responses to rewards, whereas BAS drive indexes individual sensitivity to both hedonic and motivational aspects of reward (Carver and White, 1994).

In contrast to appetizing foods, viewing disgusting or bland foods was not associated with activation in amygdala or midbrain regions. However, disgusting foods did engage areas of orbitofrontal cortex and ventral striatum. Although the striatal activation was slightly more ventral for the disgusting than appetizing foods, the two clusters of activation did overlap to some extent (supplemental Fig. 1, available at www.jneurosci.org as supplemental material). Orbitofrontal cortex has an established role in processing both rewarding and aversive/punishing stimuli (Kringelbach and Rolls, 2004), but ventral striatal involvement in aversion is a topic of debate (Mirenowicz and Schultz, 1996; Salamone et al., 1997; Horvitz, 2000; Kelley and Berridge, 2002). The activation that we observed in response to disgusting food images is consistent with recent studies that have reported ventral striatal activation to aversive stimuli, including exposure to disgusting odors (Heining et al., 2003) and the experience (Becerra et al., 2001) and anticipation (Jensen et al., 2003) of pain. However, we would not want to discount the possibility that the disgust activation incorporates Brodmann area 25 of the ventromedial prefrontal cortex, which lies ventral to the striatum and is involved in the modulation of visceral activity in response to emotional stimuli (Öngür et al., 2003).

Of additional interest, the neural response to disgusting foods in the ventral striatum and orbitofrontal cortex was correlated with reward drive. Although this might appear surprising, there is a basis in the existing literature for interpreting this relationship. The BAS-drive scale (Carver and White, 1994) was developed as a measure of drive to approach reward within the framework of Gray's (1987) "behavioral approach system." As conceived by Gray, this system is responsible for both motivating general approach behavior toward desired goals and active coping responses to aversive situations. Thus, although BAS drive is a reliable measure of drive to approach reward, it is possible that this scale may index reactivity in a broader system related to motivation to pursue and actively cope with appetizing and aversive stimuli, respectively. Indeed, recent work has shown that BAS drive is a reliable predictor of behavioral responses to facial expressions of aggression and anger-provoking situations (Carver, 2004; Putman et al., 2004).

Finally, in light of evidence showing that the fronto-striatalamygdala-midbrain network also plays an important role in drug reward (Kelley and Berridge, 2002), it is of interest that individual differences in trait reward sensitivity measures have been linked to alcohol and drug use in healthy and clinical samples (Franken, 2002; Dawe and Loxton, 2004; Knyazev, 2004; Franken et al., 2006). Hence, an important issue for future research is whether 
trait reward drive predicts activation in this network to drugrelated stimuli in drug users.

In conclusion, the results of this study demonstrate that individual differences in trait reward drive predict activation to pictures of appetizing foods in a fronto-striatal-amygdala-midbrain network implicated in food motivation and hedonics in comparative studies. Behavioral research has shown that trait reward measures predict food craving, overeating, and relative body weight (in both healthy and overweight populations), whereas neurobiological research in animals shows that pharmacological stimulation of this circuit can override satiety and cause overeating of highly palatable foods. Our study is the first to bridge the gap between these two important areas of research, providing insight into the neural mechanisms underlying appetite and the etiology of eating disorders characterized by excessive intake of food.

\section{References}

Abelson P, Kennedy D (2004) The obesity epidemic. Science 304:1413.

Anderson AK, Christoff K, Stappen I, Panitz D, Ghahremani DG, Glover G, Gabrieli JDE, Sobel N (2003) Dissociated neural representations of intensity and valence in human olfaction. Nat Neurosci 6:196-202.

Balleine BW (2005) Neural bases of food-seeking: affect, arousal and reward in corticostriatolimbic circuits. Physiol Behav 86:717-730.

Bassareo V, Di Chiara G (1999) Modulation of feeding-induced activation of mesolimbic dopamine transmission by appetitive stimuli and its relation to motivational state. Eur J Neurosci 11:4389-4397.

Becerra L, Breiter HC, Wise R, Gonzalez RG, Borsook D (2001) Reward circuitry activation by noxious thermal stimuli. Neuron 32:927-946.

Berridge KC (1996) Food reward: brain substrates of wanting and liking. Neurosci Biobehav Rev 20:1-25.

Berridge KC (2003) Pleasures of the brain. Brain Cogn 52:106-128.

Berridge KC, Robinson TE (2003) Parsing reward. Trends Neurosci 26:507-513.

Brett M, Leff AP, Rorden C, Ashburner J (2001) Spatial normalization of brain images with focal lesions using cost function masking. NeuroImage 14:486-500.

Bulik CM, Sullivan PF, Kendler KS (2003) Genetic and environmental contributions to obesity and binge eating. Int J Eat Disord 33:293-298.

Calder AJ, Lawrence AD, Young AW (2001) The neuropsychology of fear and loathing. Nat Rev Neurosci 2:352-363.

Cardinal RN, Parkinson JA, Hall J, Everitt BJ (2002) Emotion and motivation: the role of the amygdala, ventral striatum, and prefrontal cortex. Neurosci Biobehav Rev 23:321-352.

Carver CS (2004) Negative affects deriving from the behavioral approach system. Emotion 4:3-22.

Carver CS, White TL (1994) Behavioral inhibition, behavioral activation, and affective responses to impending reward and punishment: the BIS/ BAS Scales. J Pers Soc Psychol 67:319-333.

Cornell CE, Rodin J, Weingarten H (1989) Stimulus-induced eating when satiated. Physiol Behav 45:695-704.

Cusack R, Brett M, Osswald K (2003) An evaluation of the use of magnetic field maps to undistort echo-planar images. NeuroImage 18:127-142.

Davis C, Woodside DB (2002) Sensitivity to the rewarding effects of food and exercise in the eating disorders. Compr Psychiatry 43:189-194.

Davis C, Strachan S, Berkson M (2004) Sensitivity to reward: Implications for overeating and overweight. Appetite 42:131-138.

Dawe S, Loxton NJ (2004) The role of impulsivity in the development of substance use and eating disorders. Neurosci Biobehav Rev 28:343-351.

Dawe S, Gullo MJ, Loxton NJ (2004) Reward drive and rash impulsiveness as dimensions of impulsivity: implications for substance misuse. Addict Behav 29:1389-1405.

de Araujo IET, Rolls ET, Kringelbach ML, McGlone F, Phillips N (2003) Taste-olfactory convergence, and the representation of the pleasantness of flavour, in the human brain. Eur J Neurosci 18:2059-2068.

Depue RA, Collins PF (1999) Neurobiology of the structure of personality: dopamine, facilitation of incentive motivation, and extraversion. Behav Brain Sci 22:491-517.

Di Chiara G (2005) Dopamine in disturbances of food and drug motivated behavior: a case of homology? Physiol Behav 86:9-10.
Duvernoy HM (1995) The human brain stem and cerebellum. Vienna: Springer.

Duvernoy HM (1999) The human brain. Vienna: Springer.

Elliott R, Friston KJ, Dolan RJ (2000) Dissociable neural responses in human reward systems. J Neurosci 20:6159-6165.

Franken IHA (2002) Behavioral approach system (BAS) sensitivity predicts alcohol craving. Pers Individ Dif 32:349-355.

Franken IHA, Muris P (2005) Individual differences in reward sensitivity are related to food craving and relative body weight in healthy women. Appetite 45:198-201.

Franken IHA, Muris P, Georgieva I (2006) Gray's model of personality and addiction. Addict Behav 31:399-403.

Fudge JL, Breitbart MA, Danish M, Pannoni V (2005) Insular and gustatory inputs to the caudal ventral striatum in primates. J Comp Neurol 490:101-118.

Gottfried JA, O'Doherty J, Dolan RJ (2002) Appetitive and aversive olfactory learning in humans studied using event-related functional magnetic resonance imaging. J Neurosci 22:10829-10837.

Gottfried JA, O'Doherty J, Dolan RJ (2003) Encoding predictive reward value in human amygdala and orbitofrontal cortex. Science 301:1104-1107.

Gray JA (1987) The neuropsychology of emotion and personality. In: Cognitive neurochemistry (Stahl SM, Iverson SD, Goodman EC, eds), pp 171-190. Oxford: Oxford UP.

Heining M, Young AW, Ioannou G, Andrew CM, Brammer MJ, Gray JA, Phillips ML (2003) Disgusting smells activate human anterior insula and ventral striatum. Ann NY Acad Sci 1000:380-384.

Hinton EC, Parkinson JA, Holland AJ, Arana FS, Roberts AC, Owen AM (2004) Neural contributions to the motivational control of appetite in humans. Eur J Neurosci 20:1411-1418.

Horvitz JC (2000) Mesolimbocortical and nigrostriatal dopamine responses to salient non-reward events. Neuroscience 96:651-656.

Ikemoto S, Panksepp J (1999) The role of nucleus accumbens dopamine in motivated behavior: a unifying interpretation with special reference to reward-seeking. Brain Res Brain Res Rev 31:6-41.

Jensen J, McIntosh AR, Crawley AP, Mikulis DJ, Remington G, Kapur S (2003) Direct activation of the ventral striatum in anticipation of aversive stimuli. Neuron 40:1251-1257.

Jorm AF, Christensen H, Henderson AS, Jacomb PA, Korten AE, Rodgers B (1998) Using the BIS/BAS scales to measure behavioural inhibition and behavioural activation: factor structure, validity and norms in a large community sample. Pers Individ Dif 26:49-58.

Kelley AE (2004) Ventral striatal control of appetitive motivation: role in ingestive behavior and reward-related learning. Neurosci Biobehav Rev 27:765-776.

Kelley AE, Berridge KC (2002) The neuroscience of natural rewards: relevance to addictive drugs. J Neurosci 22:3306-3311.

Kelley AE, Baldo BA, Pratt WE, Will MJ (2005) Corticostriatalhypothalamic circuitry and food motivation: integration of energy, action and reward. Physiol Behav 86:773-795.

Killgore WDS, Young AD, Femia LA, Bogorodzki P, Rogowska J, YurgelunTodd DA (2003) Cortical and limbic activation during viewing of highversus low-calorie foods. NeuroImage 19:1381-1394.

Knyazev GG (2004) Behavioural activation as predictor of substance use: mediating and moderating role of attitudes and social relationships. Drug Alcohol Depend 75:309-321.

Kringelbach ML, Rolls ET (2004) The functional neuroanatomy of the human orbitofrontal cortex: evidence from neuroimaging and neuropsychology. Prog Neurobiol 72:341-372.

Kringelbach ML, O’Doherty J, Rolls ET, Andrews C (2003) Activation of the human orbitofrontal cortex to a liquid food stimulus is correlated with its subjective pleasantness. Cereb Cortex 13:1064-1071.

LaBar KS, Gitelman DR, Parrish TB, Kim Y-H, Nobre AC, Mesulam M-M (2001) Hunger selectively modulates corticolimbic activation to food stimuli in humans. Behav Neurosci 115:493-500.

McAlonan GM, Robbins TW, Everitt BJ (1993) Effects of medial dorsal thalamic and ventral pallidal lesions on the acquisition of a conditioned place preference: further evidence for the involvement of the ventral striatopallidal system in reward-related processes. Neuroscience 52:605-620.

Mirenowicz J, Schultz W (1996) Preferential activation of midbrain dopamine neurons by appetitive rather than aversive stimuli. Nature 379:449-451. 
Morris JS, Dolan RJ (2001) Involvement of human amygdala and orbitofrontal cortex in hunger-enhanced memory for food stimuli. J Neurosci 21:5304-5310.

O’Doherty JP, Deichmann R, Critchley HD, Dolan RJ (2002) Neural responses during anticipation of a primary taste reward. Neuron 33:815-826.

Öngür D, Price JL (2000) The organization of networks within the orbital and medial prefrontal cortex of rats, monkeys and humans. Cereb Cortex 10:206-219.

Öngür D, Ferry AT, Price JL (2003) Architectonic subdivision of the human orbital and medial prefrontal cortex. J Comp Neurol 460:425-449.

Pecina S, Berridge KC (2000) Opioid site in nucleus accumbens shell mediates eating and hedonic "liking" for food: map based on microinjection Fos plumes. Brain Res 863:71-86.

Petrovich GD, Setlow B, Holland PC, Gallagher M (2002) Amygdalohypothalamic circuit allows learned cues to override satiety and promote eating. J Neurosci 22:8748-8753.

Pickering AD, Gray JA (1999) The neuroscience of personality. In: Handbook of personality: theory and research, Ed 2 (Pervin LA, John OP, eds), pp 277-299. New York: Guilford.

Putman P, Hermans E, van Honk J (2004) Emotional Stroop performance for masked angry faces: it's BAS, not BIS. Emotion 4:305-311.

Rolls ET, Kringelbach ML, de Araujo IET (2003) Different representations of pleasant and unpleasant odours in the human brain. Eur J Neurosci 18:695-703.

Ross SR, Millis SR, Bonebright TL, Bailley SE (2002) Confirmatory factor analysis of the behavioral inhibition and activation scales. Pers Individ Dif 33:861-865.
Salamone JD, Cousins MS, Snyder BJ (1997) Behavioral functions of nucleus accumbens dopamine: empirical and conceptual problems with the anhedonia hypothesis. Neurosci Biobehav Rev 21:341-359.

Saper CB, Chou TC, Elmquist JK (2002) The need to feed: homeostatic and hedonic control of eating. Neuron 36:199-211.

Small DM, Zatorre RJ, Dagher A, Evans AC, Jones-Gotman M (2001) Changes in brain activity related to eating chocolate: from pleasure to aversion. Brain 124:1720-1733.

Smith KS, Berridge KC (2005) The ventral pallidum and hedonic reward: neurochemical maps of sucrose "liking" and food intake. J Neurosc 25:8637-8649.

Tiggemann M, Kemps E (2005) The phenomenology of food cravings: the role of mental imagery. Appetite 45:305-313.

Tindell AJ, Berridge KC, Aldridge JW (2004) Ventral pallidal representation of pavlovian cues and reward: population and rate codes. J Neurosci 24:1058-1069.

Toates FM (1981) The control of ingestive behaviour by internal and external stimuli: a theoretical review. Appetite 2:35-50.

Tzourio-Mazoyer N, Landeau B, Papathanassiou D, Crivello F, Etard O, Delcroix N, Mazoyer B, Joliot M (2002) Automated anatomical labeling of activations in SPM using a macroscopic anatomical parcellation of the MNI MRI single-subject brain. NeuroImage 15:273-289.

Wang G-J, Volkow ND, Telang F, Jayne M, Ma J, Rao M, Zhu W, Wong CT, Pappas NR, Geliebter, Fowler JS (2004) Exposure to appetitive food stimuli markedly activates the human brain. NeuroImage 21:1790-1797.

Zald DH (2003) The human amygdala and the emotional evaluation of sensory stimuli. Brain Res Brain Res Rev 41:88-123. 